\title{
Impact of lifestyle in severe psychiatric disorders and brain morphology
}

\author{
Andrea Schmitt • Peter Falkai
}

Published online: 27 July 2013

(C) Springer-Verlag Berlin Heidelberg 2013

During the last decades, physical exercise has been part of treatment strategies in psychiatric hospitals, but to date there is only sparse information on the effectivity, standardized protocols are missing. Invited to investigate potential influences of exercise on schizophrenia and affective disorders, Malchow et al. [1] in their important review looked at respective studies reaching back to the early 1990s, according to which physical exercise very well might positively influence hippocampus volume and cognitive impairment via stimulating neurogenesis, synaptogenesis and neurotransmission in healthy and affected persons. Since cognitive impairment is known to negatively influence both patient's quality of life and their ability to hold down a job, these results allow the conclusion that modern therapeutic approaches should comprise physical exercise as add-on of a multimodal intervention program in severe psychiatric diseases. Falkai et al. [2] again investigated the effects of aerobic exercise on brain plasticity in a small sample of chronic schizophrenia patients and healthy controls. After 3 months, exercise yielded gray matter density that increases in the right frontal and occipital cortex merely in healthy probands. This may lead to the assumption that in contrast to the hippocampus, synaptic plasticity is compromised in the cortex of schizophrenia patients, probably due to the effects of disease or antipsychotic medication. However, a replication study in a larger sample is under way and could perhaps lead to the detection of comparable effects in patients. Brain plasticity at least seems to be effectively

A. Schmitt $(\bowtie) \cdot$ P. Falkai

Department of Psychiatry and Psychotherapy,

Ludwig-Maximilians-University Munich,

Nußbaumstr. 7, 80336 Munich, Germany

e-mail: Andrea.Schmitt@med.uni-muenchen.de susceptible to specific conditions like low-frequency repetitive transcranial magnetic stimulation compared with standard rTMS, as Klirova et al. [3] show in a study with patients suffering from auditory hallucinations, even though this study also bases upon a relatively small patient's sample.

In a molecular approach on schizophrenia neuropathology, Williams et al. [4] undertook a post-mortem investigation of neuropathological changes in the so far not thoroughly examined nucleus basalis in 41 patients and age-matched controls. Via coronal nucleus basalis sections, the authors detected significant glial cell abnormalities with possible impacts on cortical limbic and subcortical areas that could be a hint on cortical limbic disturbances and limbic dysfunction in schizophrenia. Schönknecht et al. [5] in turn substituted post-mortem for in vivo studies and mapped the hypothalamus and its subdivisions, which are known to be involved, e.g., in schizophrenia or affective disorders, using T1-weighted and diffusion-weighted DTI scans in healthy volunteers. The group thus was able to manually delineate an individual mask of the hypothalamus in each subject and to compare the microstructural hypothalamic parcellations to the respective regions reported in the postmortem studies. This opportunity of structurally analyzing hypothalamic subnuclei in vivo might contribute to better understand the pathogenesis of neuropsychiatric conditions.

Imaging studies on reward processing in schizophrenia patients and healthy controls have shown differential activations of dopaminergic brain areas to be associated with adaptive changes in response to speed related to different reward values. Lernbass et al. [6] now investigated this schizophrenia-associated dysfunction on the behavioral level in 49 medicated patients and 49 controls, which confirmed the reported deficits in reward processing in the 
patient group. Moreover, they found the effect to be more pronounced in a subgroup of nonsmoking patients who additionally showed a positive correlation with medication type and with the PANSS score, for which reason the authors assume that smoking modulates medication-associated side effects on reward processing in schizophrenia. The effects of nicotine were also subject of the study by Drusch et al. [7] who focussed on social cognition, social competence and subjective stress in schizophrenia patients and healthy controls. Being administered either nicotine or placebo after $24 \mathrm{~h}$ of smoking, abstinence patients and controls surprisingly did not differ in performing a facial affect recognition task, whereas in a role-play situation, healthy controls showed more nonverbal expressions and had a lower subjective stress level than schizophrenia patients. Contradictory to the hypothesized development of nicotine dependence in more than $80 \%$ of schizophrenia patients as self-medication of cognitive deficits and increased vulnerability to stress, the authors did not find significant effects of nicotine which they ascribe to the dosage used and delay between nicotine administration and performance of the role-play task.

Lotzin et al. [8] investigated whether the impairment of facial expressions during social interaction with adult schizophrenia patients would also be identifiable in adolescent-onset patients. The group assessed facial expressions of emotions and speaker position in adolescent schizophrenia patients and matched healthy controls during a problem-solving discussion with their primary caregivers. Coding the facial emotions with the Emotional Facial Action Coding System, the authors found significantly lower rates per minute of expressing positive emotions in the patients group, whereas the rate of expressions indicating negative emotions did not deviate significantly from controls. However, negative facial expressions in the control group were related to speaking, but no such relationship could be observed in the patient group indicating an emotion-specific impairment of positive facial expressions plus their deviant relation to speech in the early-onset patients.

\section{References}

1. Malchow B, Reich-Erkelenz D, Oertel-Knöchel V, Keller K, Hasan A, Schmitt A, Scheewe TW, Cahn W, Kahn RS, Falkai P (2013) The effects of physical exercise in schizophrenia and affective disorders. Eur Arch Psychiatry Clin Neurosci. doi:10. 1007/s00406-013-0423-2

2. Falkai P, Malchow B, Wobrock T, Gruber O, Schmitt A, Honer WG, Pajonk F-G, Sun F, Cannon TD (2012) The effect of aerobic exercise on cortical architecture in patients with chronic schizophrenia: a randomized controlled MRI study. Eur Arch Psychiatry Clin Neurosci. doi:10.1007/s00406-012-0383-y

3. Klirova M, Horacek J, Novak T, Cermak J, Spaniel F, Skrdlantova L, Mohr P, Höschl C (2012) Individualized rTMS neuronavigated according to regional brain metabolism (18FGD PET) has better treatment effects on auditory hallucinations than standard positioning of rTMS: a double-blind, sham-controlled study. Eur Arch Psychiatry Clin Neurosci. doi:10.1007/s00406-012-0368-x

4. Williams MR, Marsh R, Macdonald CD, Jain J, Pearce RKB, Hirsch SR, Ansorge O, Gentleman SM, Maier M (2012) Neuropathological changes in the nucleus basalis in schizophrenia. Eur Arch Psychiatry Clin Neurosci. doi:10.1007/s00406-012-0387-7

5. Schönknecht P, Anwander A, Petzold F, Schindler S, Knösche TR, Möller HE, Hegerl U, Turner R, Geyer S (2012) Diffusion imaging-based subdivision of the human hypothalamus: a magnetic resonance study with clinical implications. Eur Arch Psychiatry Clin Neurosci. doi:10.1007/s00406-012-0389-5

6. Lernbass B, Grön G, Wolf ND, Abler B (2012) Cigarette smoking modulates medication-associated deficits in a monetary reward task in patients with schizophrenia. Eur Arch Psychiatry Clin Neurosci. doi:10.1007/s00406-012-0370-3

7. Drusch K, Lowe A, Fisahn K, Brinkmeyer J, Musso F, Mobascher A, Warbrick T, Shah J, Ohmann C, Winterer G, Wölwer W (2012) Effects of nicotine on social cognition, social competence and selfreported stress in schizophrenia patients and healthy controls. Eur Arch Psychiatry Clin Neurosci. doi:10.1007/s00406-012-0377-9

8. Lotzin A, Haack-Dees B, Resch F, Romer G, Ramsauer B (2012) Facial emotional expression in schizophrenia adolescents during verbal interaction with a parent. Eur Arch Psychiatry Clin Neurosci. doi:10.1007/s00406-012-0386-8 